\title{
Significance of Serum IL-2, IL-6 and IL-8 Detection in Patients with Hyperthyroidism and Subclinical Hyperthyroidism
}

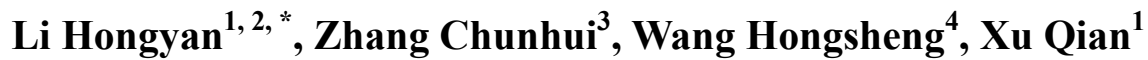 \\ ${ }^{1}$ Department of Medical Technology, Cangzhou Medical College, Cangzhou, China \\ ${ }^{2}$ Cangzhou Thyroid Disease Engineering Research Center, Cangzhou, China \\ ${ }^{3}$ Department of Blood Transfusion of Hejian People's Hospital, Cangzhou, China \\ ${ }^{4}$ Thyroid Diagnosis and Treatment Center of Cangzhou Traditional Chinese Medicine-Western Hospital, Cangzhou, China
}

Email address:

czyzjyk@126.com (Li Hongyan)

${ }^{*}$ Corresponding author

\section{To cite this article:}

Li Hongyan, Zhang Chunhui, Wang Hongsheng, Xu Qian. Significance of Serum IL-2, IL-6 and IL-8 Detection in Patients with Hyperthyroidism and Subclinical Hyperthyroidism. Science Journal of Public Health. Vol. 7, No. 5, 2019, pp. 159-162. doi: $10.11648 /$ j.sjph.20190705.14

Received: July 22, 2019; Accepted: September 25, 2019; Published: September 27, 2019

\begin{abstract}
Objective: This paper is to explore the relationship between IL-2, IL-6, IL-8 and the development of hyperthyroidism and subclinical hyperthyroidism. Methods The serum levels of IL-2, IL-6 and IL-8 were determined in 251 cases of hyperthyroidism, 242 cases of subclinical hyperthyroidism and 130 cases of normal control. Results The level of IL-2 in hyperthyroidism group was significantly lower than that in the control group $(\mathrm{P}<0.01)$, and the levels of IL-6 and IL-8 were significantly higher than those in the control group $(\mathrm{P}<0.01)$; The level of IL-2 in subclinical hyperthyroidism group was lower than that in the control group $(\mathrm{P}<0.05)$, and the levels of IL- 6 and IL-8 were higher than those in the control group $(\mathrm{P}<0.05)$. The level of IL-2 in hyperthyroidism group was lower than that in subclinical hyperthyroidism group, and the level of IL-6 and IL-8 were higher than those in subclinical hyperthyroidism group. The two groups of IL-2 were negatively correlated with serum FT3, FT 4, TT3, and TT4, and IL-6 and IL-8 were positively correlated with serum FT3, FT 4, TT3, and TT4. Conclusions IL-2, IL-6, and IL-8 all participated in the development process of hyperthyroidism and subclinical hypothyroidism, which contributed to the early discovery of subclinical hyperthyroidism.
\end{abstract}

Keywords: Hypothyroidism, Subclinical Hypothyroidism, IL-2, IL-6, IL-8

\section{Introduction}

Cangzhou is a high iodine excess area because of its water, thus with relatively high incidence of thyroid diseases. As an endemic disease in Cangzhou and its surrounding areas, hyperthyroidism (hyperthyroidism) has attracted much attention for many years. Hyperthyroidism refers to thyrotoxicosis caused by excessive production of thyroid hormones in the thyroid gland itself. Patients with hyperthyroidism are not only having clinical manifestations such as increased excitability of the circulatory, digestive and nervous systems, and hypermetabolism, etc, but also appearing in many parts of the body tissue and organ, lesions, especially and more commonly cardiovascular and nervous system diseases [1]. Subclinical Hypothyroidism is a special manifestation of hyperthyroidism, patients with such a disease would have mild symptoms, and are easily neglected during the examination [2]. However, it is possible that this disease may develop into clinical hyperthyroidism [3], and increase the risk of cardiac death $[4,5]$. Therefore, early diagnosis and timely intervention of hyperthyroidism can greatly reduce the cost of treatment and the complications. Over $85 \%$ of hyperthyroidism is caused by Graves disease, which is an autoimmune disease [6].

In my research, the changes of serum immunoregulatory factors IL-2, IL-6 and IL-8 levels in patients with hyperthyroidism and subclinical hyperthyroidism in 
water-source-caused iodine excess areas were measured to explore the relationship between them and the occurrence and development of hyperthyroidism and subclinical hypothyroidism, to provide the basis for the study, diagnosis and treatment of the pathogenesis of hyperthyroidism and subclinical hyperthyroidism, and to provide $\mathrm{r}$.

\section{Datas and methods}

\subsection{Clinical Datas}

From March 2016 to October 2018, 493 patients (194 males and 299 females) were divided into two groups: hyperthyroidism group ( 251 cases, 98 males and 153 females, aged 21 to 70 years old, averaging $42.2 \pm 15.1$ years old)) and the Subclinical hyperthyroidism group (242 cases, 95 males and 147 females, averaging $46.5 \pm 17.4$ years old). All cases were newly diagnosed cases, without any treatment, and diagnosed by clinic and laboratory to exclude the possible endocrine system and non-thyroid systemic diseases.

At the same time, 130 healthy people ( 58 males, 72 females, age $21-70$, averaging $45.3 \pm 12.5$ years old) were selected as normal control group, endocrine system and non-thyroid systemic diseases excluded, heart, liver, kidney, lung and other important organs confirmed normal, and Thyroid function and blood routine examination confirmed being normal.

In this research, all subjects or their family members gave their informed consent, signed their informed consent forms, and approval of the Ethics Committee gained.

\subsection{Methods}

Researching Methods: After receiving a fasting hemospasia in early morning, $4 \mathrm{ml}$ of venous blood was collected from the patients and healthy people, then rest it at room temperature for $1-2 \mathrm{~h}$, after that, the serum was separated by high-speed centrifugation and stored in refrigerator at $-40^{\circ} \mathrm{C}$ for measurement. The five indexes of serum thyroid function: free triiodothyronine (FT3), free thyroxine (FT4), total triiodothyronine (TT3), total thyroxine (TT4) and thyroid stimulating hormone (TSH) were determined by automatic electrochemistry of Cobas e602 and its supporting reagents from Roche Company of Germany. Immunoregulatory factors IL-2, IL-6, IL-8 were determined by Enzyme-Linked Immunosorbnent Assay method, Operated strictly in accordance with the instructions and results reported with $\mathrm{pg} / \mathrm{ml}$.

\subsection{Diagnostic Standard}

The diagnostic standard for hyperthyroidism and Subclinical hyperthyroidism hypothyroidism were based on the Chinese guidelines for diagnosis and treatment of thyroid Diseases of 2008. (1) hyperthyroidism: Have the symptoms or signs of hyperthyroidism, and conforms with FT3 $>7.1 \mathrm{pmol} / \mathrm{L}$, FT4 $>22 \mathrm{pmol} / \mathrm{L}, \quad \mathrm{TSH}<0.27 \mathrm{mIU} / \mathrm{L} ; \quad$ (2) Subclinical hyperthyroidism: TSH $<0.27 \mathrm{~m} \mathrm{IU/L,} \mathrm{FT3,} \mathrm{FT4} \mathrm{are} \mathrm{within} \mathrm{the}$ normal range.

\subsection{Statistical Analysis}

SPSS19.0 statistical software was used to process the data, the metrological data were expressed as $\overline{\mathrm{x}}_{ \pm \mathrm{s}}$, the quantitative data were compared by t-test, and the correlation among the factors was analyzed by linear correlation analysis. $\mathrm{P}<0.05$ suggests that there were significant differences.

\section{Results}

\subsection{Comparison of the Levels of Serum IL-2, IL-6 and IL-8 in All the Groups}

Compared with the normal control group, the levels of IL-2 of the hyperthyroidism group were significantly lower $(\mathrm{P}<0.01)$, while the levels of IL-6 and IL-8 were significantly higher $(\mathrm{P}<0.01)$; the levels of IL-2 of the Subclinical hyperthyroidism group was decreased, and IL-6 and IL-8 increased $(\mathrm{P}<0.05)$. The level of IL-2 in hyperthyroidism group was lower than that in Subclinical hyperthyroidism group, while the levels of IL-6 and IL- 8 were higher than that in hyperthyroidism group $(\mathrm{P}<0.05)$ (Table 1$)$.

Table 1. The levels of serum $I L-2, I L-6$ and $I L-8$ in all the groups $(x \pm s)$.

\begin{tabular}{llll}
\hline groups & control group $(\mathbf{n = 1 3 0})$ & hyperthyroidism group $(\mathbf{n = 2 5 1})$ & Subclinical hyperthyroidism group $(\mathbf{n = 2 4 2})$ \\
\hline IL-2 $(\mathrm{pg} / \mathrm{ml})$ & $476.1 \pm 35.6$ & $172.4 \pm 24.8$ & $255.6 \pm 27.1$ \\
IL-6 $(\mathrm{pg} / \mathrm{ml})$ & $1.3 \pm 0.1$ & $2.7 \pm 0.2$ & $2.1 \pm 0.1$ \\
IL-8 $(\mathrm{pg} / \mathrm{ml})$ & $60.4 \pm 13.7$ & $90.4 \pm 16.5$ & $74.6 \pm 18.6$ \\
\hline
\end{tabular}

\subsection{Analysis of Correlation of Serum $I L-2, I L-6, I L-8$ and Thyroid Function Indexes in Hyperthyroidism Group}

Table 2. Analysis of Correlation of serum $I L-2, I L-6, I L-8$ and thyroid function indexes in hyperthyroidism group.

\begin{tabular}{llllll}
\hline & $\mathbf{F T}_{\mathbf{3}}$ & $\mathbf{F T}_{\mathbf{4}}$ & $\mathbf{T T}_{\mathbf{3}}$ & $\mathbf{T T}_{\mathbf{4}}$ & $\mathbf{T S H}$ \\
\hline \multirow{2}{*}{ IL-2 } & $\mathrm{r}=-0.801$ & $\mathrm{r}=-0.615$ & $\mathrm{r}=-0.457$ & $\mathrm{r}=-0.413$ & $\mathrm{r}=0.635$ \\
& $P=0.012$ & $P=0.004$ & $P=0.015$ & $P=0.005$ & $P=0.151$ \\
IL-6 & $\mathrm{r}=0.521$ & $\mathrm{r}=0.427$ & $\mathrm{r}=0.391$ & $\mathrm{r}=0.214$ & $\mathrm{r}=-0.196$ \\
& $P=0.014$ & $P=0.009$ & $P=0.024$ & $P=0.019$ & $P=0.013$ \\
IL-8 & $\mathrm{r}=0.716$ & $\mathrm{r}=0.742$ & $\mathrm{r}=0.419$ & $\mathrm{r}=0.406$ & $\mathrm{r}=0.471$ \\
& $P=0.011$ & $P=0.014$ & $P=0.002$ & $P=0.006$ & $P=0.393$ \\
\hline
\end{tabular}

Correlation analysis of serum IL-2, IL-6, IL-8 and thyroid function indexes in hyperthyroidism group, IL-2 was negatively correlated with serum FT3, FT4, TT3, TT4, meanwhile, IL-6, IL-8 positively correlated with blood Serum FT3, FT4, TT3 and TT4, and IL-6 was negatively correlated with serum TSH (Table 2).

\subsection{Analysis of Correlation of Serum IL-2, IL-6, IL-8 and Thyroid Function Indexes in Subclinical Hyperthyroidism Group}

Correlation analysis of serum IL-2, IL-6, IL-8 and thyroid 
function indexes in Subclinical hyperthyroidism group, IL-2 was negatively correlated with serum FT3, FT4, TT3, TT4, meanwhile, IL-6, IL-8 positively correlated with blood Serum FT3, FT4, TT3 and TT4, and IL-6 was negatively correlated with serum TSH (Table 3).

Table 3. Analysis of Correlation of serum IL-2, IL-6, IL-8 and thyroid function indexes in Subclinical hyperthyroidism group.

\begin{tabular}{llllll}
\hline & $\mathbf{F T}_{3}$ & $\mathbf{F T}_{\mathbf{4}}$ & TT $_{3}$ & TT $_{4}$ & TSH \\
\hline \multirow{2}{*}{ IL-2 } & $\mathrm{r}=-0.521$ & $\mathrm{r}=-0.315$ & $\mathrm{r}=-0.247$ & $\mathrm{r}=-0.218$ & $\mathrm{r}=0.364$ \\
& $P=0.023$ & $P=0.025$ & $P=0.014$ & $P=0.018$ & $P=0.162$ \\
IL-6 & $\mathrm{r}=0.461$ & $\mathrm{r}=0.367$ & $\mathrm{r}=0.292$ & $\mathrm{r}=0.222$ & $\mathrm{r}=-0.181$ \\
& $P=0.011$ & $P=0.008$ & $P=0.016$ & $P=0.009$ & $P=0.010$ \\
IL-8 & $\mathrm{r}=0.492$ & $\mathrm{r}=0.362$ & $\mathrm{r}=0.319$ & $\mathrm{r}=0.413$ & $\mathrm{r}=0.331$ \\
& $P=0.009$ & $P=0.010$ & $P=0.006$ & $P=0.016$ & $P=0.437$ \\
\hline
\end{tabular}

\section{Discussions}

IL-2, IL-6 and IL-8 are multifunctional cytokines produced by lymphocyte and participate in a variety of biological effects IL-2 is mainly produced by Th1 cells, involved in the regulation of lymphocyte balance and function, and is an important cytokine involved in cellular immunity, could be used as an important indicator of the function of body's cellular immune [7].

IL-6 is the representative of Th2 type cytokine, which is an early inflammatory factor [8] and plays an important role in humoral immune response. IL-8 is a multi-derived cytokine, which is produced by many kinds of cells, such as vascular endothelial cells, monocytes, T lymphocytes and so on. It has a strong chemotactic effect on specific and non-specific immunoreactive cells [9], thus affecting the function of immunoreactive cells such as B lymphocytes, prompting the body to produce autoantibodies and accelerating the occurrence of hyperthyroidism. Cytokine is the center of biomedical research [10]. For many years, the changes of cytokine in thyroid diseases have been differently reported. Zhang Yunming et al [11] reported that the levels of serum IL-6 and TNF- $\alpha$ in patients with GD increased significantly before treatment, and decreased significantly after treatment $^{131}$, while IL-2 decreased significantly before treatment and increased gradually after treatment. Serum levels of IL-6 and TNF- $\alpha$ were positively correlated with FT3 and FT4, and IL-2 was negatively correlated with FT3 and FT4. Gao Haibo et al [12] found that the levels of IL-2 and IL-6 were significantly increased in patients with GD before treatment, but there was no significant correlation with the levels of FT3 and FT4. The study of Wang Xiaoying et al [13] showed that the level of IL-6 in patients with GD was higher than that in the control group before treatment, but there was no significant difference, but it was positively correlated with FT3 and FT4. After treatment, the level of IL-6 decreased gradually. Shen Ji et al [14] also found that IL-6 in patients with GD increased significantly before treatment and decreased significantly after treatment. Wei Feng et al [15] reported that IL-8 increased in the hyperthyroidism, which participated in the autoimmune and inflammatory reaction of patients with hyperthyroidism.
My research shows that the levels of IL-2 in hyperthyroidism group and Subclinical hypothyroidism group were decreased, and are negatively correlated with serum FT3, FT4, TT3 and TT4; while the levels of IL-6 and IL-8 are increased, and are positively correlated with serum FT3, FT4, TT3 and TT4, and IL-6 negatively correlated with serum TSH. The changes of IL-2, IL-6 and IL-8 are caused by $\mathrm{Th} 1 / \mathrm{Th} 2$ equilibrium drift [16]. Under normal condition, Th1 and Th2 cells were in dynamic equilibrium, and the cellular and humoral immune responses functions were normal. Once the cellular immunity or humoral immunity is abnormal, the balance of Th1 and Th2 cells is destroyed, and the cytokines produced by them would also be changed. When humoral immunity is dominated by Th2-type cytokines, the body produces a large amount of thyroid excitatory antibodies; this would stimulate the synthesis of thyroid hormone [17], causing thyroid dysfunction, and resulted in hyperthyroidism. Also, thyroid dysfunction would make the immune damage worse, increase the production or consumption of cytokines, as a result of which, the thyroid dysfunction becomes worse.

\section{Conclusion}

These results suggest that IL-2, IL- 6 and IL- 8 are involved in the occurrence and development of hyperthyroidism and subclinical hyperthyroidism, and they regulate and influence each other, which, to some extent, can reflect the changes of thyroid function. It is of great reference value to study the pathogenesis of thyroid disease, to judge the prognosis of thyroid function and to evaluate the clinical condition of thyroid disease. The level of IL-2 in hyperthyroidism group was lower than that in Subclinical hyperthyroidism group, while the levels of IL-6 and IL-8 were higher in hyperthyroidism group than that in Subclinical hyperthyroidism group, which suggested that these three indexes are of great significance for early detection, as well as timely and effective intervention of hyperthyroidism, and could be an early predictor of the development of hypothyroidism. In spite of this, the results of each scholar are not the same, further research is needed to see whether the iodine nutrition status of the selected cases, the methods used in testing, and other reasons are related.

\section{Fund Project}

Self-financed Project of Hebei Science and Technology Research and Development Program (No. 162777205).

Cangzhou Science and Technology Research and Development Guidance Program Project (Ref. 151302011).

\section{References}

[1] Hou Zhenjiang, Zhang Jingyu, Bian Yunzhuo, et al. Changes of serum lipid level, its ratio and cystatin $\mathrm{C}$ level in patients with hyperthyroidism and subclinical hyperthyroidism [J]. Shandong Medical Journal, 2017, 57 (39): 91-93. 
[2] Yu Tao. Dose effect of compound hyperthyroidism tablet in treatment of subclinical hyperthyroidism [J]. China Medicine and Pharmacy, 2017, 7 (2): 34-36.

[3] Lu Zaiying, Zhong nanshan. Internal Medicine [M]. Beijing 7th Edition: People's Health Publishing House, 2012: 714.

[4] Collet TH, Gussekloo J, Bauer DC, et al. Subclinical hyperthyroidism and the risk of coronary heart disease and mortality [J]. Arch Intern Med, 2012, 172 (10): 799-809.

[5] Xu Xiaojin, Geng Jin, Hu Ting-ting, et al. Effect of subclinical hyperthyroidism on adverse cardiovascular events in patients with type 2 diabetes mellitus [J]. Shandong Medical Journal, 2017, 57 (14): 68-71.

[6] Pang Aiping. The characteristics of patients with Graves disease complicated with hyperthyroidism heart disease and the clinical significance of related biochemical indicators [D]. Guangxi Medical University, 2018.

[7] Wu Yajun, Huang Yong-de. Peptic ulcer with Hp infection and its relationship with serum IL-6, IL-8 and IL-2 [J]. Clinical Journal of Medical Officers, 2017, 45 (01): 38-41.

[8] Li-Chao, Guo Qi. Changes of Serum IL-1 $\beta$, IL-2, IL-6, IL-8 and TNF- $\alpha$ Levels in patients with epilepsy and clinical significance $[\mathrm{J}]$. Journal of Preventive Medicine of Chinese People's Liberation Army, 2018, 36 (03): 375-377+385.

[9] Ma Xiaolan, Wu Xuecheng, Zhang Shiyi. Observation of serum TNF, IL-6 and IL-8 levels in patients with hyperthyroidism and hypothyroidism [J]. Journal of Radioimmunology, 2002, 15 (3): 166.

[10] Guo Jianlian, Liu Huina, Lu Long-kun, et al. The relationship between the levels of serum IL-2, IL- 6 , IL-8, TNF- $\alpha$ and bloodstream infection in critically ill patients $[\mathrm{J}]$. Current Immunology, 2018, 38 (02): 120-123.

[11] Zhang Yunming, Song Mingqiang. Changes of IL-2, IL-6, TNF concentration after 131I therapy in patients with hyperthyroidism from Graves' disease [J]. Guide of China Medicine, 2013, 11 (2): 8-9.

[12] Gao Haibo, Zhang Yawei, Hu Jiankang, et al. Effect of IL-2 and IL-6 levels on prognosis of 131 iodine treatment in Graves' patients [J]. Acta Medicinae Universitatis Scientiae et Technologiae Huazhong, 2016, (6): 716-718.

[13] Wang Xiaoying, Gaolin, Liao Xin. et al. Relevance study between serum levels of Visfatin, IL-6 and TNF-alpha and thyroid function in patients with Graves' disease [J]. Guizhou Medical Journal, 2018, 42 (02: 162-163).

[14] Shen J, Li Z, Li W, et a1. Thl, Th2, and Th17 cytokine in-volvement in thyroid associated ophthalmopathy [J]. DisMarkers, 2015, 10 (7): 638-643.

[15] Wei-Feng, Jiao Yanxiang, Guang Yan Qin, et al. Influence of different thyroid functional statuses on human serum IL-8, TNF Levels [J]. Journal of Radioimmunology, 2003 (04): 193-195.

[16] Zhao Jiwei, Yang Lijuan, Sun Xuefeng. Advances in the study of the relationship between thyroid tumors and cytokines IL-4, IL-10, IFN-gamma and IL-17 [J]. Journal of Hebei North University (Natural Science Edition), 2015, 31 (3): 102-105.

[17] Wang Junfang, Zhang Wei, Li Jing-li, et al. Effect of selenium on serum levels of TPOAb and TGAb in patients with Graves' disease [J]. Chinese Journal of Integrative Medicine on Cardio-Cerebrovascular Disease, 2013, 11 (04): 502-503. 